\title{
THE EXTENDED STELLAR COMPONENT OF GALAXIES THE NATURE OF DARK MATTER
}

\author{
C. Power and A. S. G. Robotham
}

International Centre for Radio Astronomy Research, University of Western Australia, 35 Stirling Highway, Crawley, WA 6009, Australia; chris.power@icrar.org Received 2016 March 23; revised 2016 May 16; accepted 2016 May 16; published 2016 June 27

\begin{abstract}
Deep observations of galaxies reveal faint extended stellar components (hereafter ESCs) of streams, shells, and halos. These are a natural prediction of hierarchical galaxy formation, as accreted satellite galaxies are tidally disrupted by their host. We investigate whether or not global properties of the ESC could be used to test of dark matter, reasoning that they should be sensitive to the abundance of low-mass satellites, and therefore the underlying dark matter model. Using cosmological simulations of galaxy formation in the favored Cold Dark Matter (CDM) and Warm Dark Matter (WDM) models $\left(m_{\mathrm{WDM}}=0.5,1,2 \mathrm{keV} / c^{2}\right)$, which suppress the abundance of low-mass satellites, we find that the kinematics and orbital structure of the ESC is consistent across models. However, we find striking differences in its spatial structure, as anticipated-a factor of $\sim 10$ drop in spherically averaged mass density between $\sim 10 \%$ and $\sim 75 \%$ of the virial radius in the more extreme WDM runs $\left(m_{\mathrm{WDM}}=0.5,1 \mathrm{keV} / c^{2}\right)$ relative to the CDM run. These differences are consistent with the mass assembly histories of the different components, and are present across redshifts. However, even the least discrepant of the WDM models is incompatible with current observational limits on $m_{\mathrm{WDM}}$. Importantly, the differences we observe when varying the underlying dark matter are comparable to the galaxy-to-galaxy variation we expect within a fixed dark matter model. This suggests that it will be challenging to place limits on dark matter using only the unresolved spatial structure of the the ESC.
\end{abstract}

Key words: dark matter - galaxies: evolution - galaxies: formation - methods: numerical

\section{INTRODUCTION}

Arguably the defining prediction of the canonical Cold Dark Matter (CDM) model of cosmological structure formation is that dark matter haloes should contain an abundance of lowmass substructure haloes (hereafter subhalos; e.g., Klypin et al. 1999; Moore et al. 1999; Reed et al. 2005; Springel et al. 2008; Ishiyama et al. 2013), remnants of the merging hierarchy through which their hosts assembled. Cosmological $\mathrm{N}$-body simulations have revealed that the number density of these subhalos increases with decreasing subhalo mass scale (e.g., Reed et al. 2005), approximately as $n_{\text {sub }} \propto M_{\text {sub }}^{-\alpha}$, with $\alpha \simeq 1.9$ (e.g., Gao et al. 2004; Diemand et al. 2007; Springel et al. 2008; Garrison-Kimmel et al. 2014), and this applies equally in the dark matter host halos of massive galaxy clusters of $10^{15} M_{\odot}$ down to the hosts of galaxies like the Milky Way $\left(\sim 10^{12} M_{\odot}\right)$ and dwarfs (e.g., Angulo et al. 2009; Xie \& Gao 2015; Rodriguez-Puebla et al. 2016).

Alternatives to the CDM model, such as Warm Dark Matter (WDM), suppress the abundance of low-mass dark matter halos, and consequently the abundance of subhalos, (e.g., Smith \& Markovic 2011; Benson et al. 2013; Pacucci et al. 2013; Schneider et al. 2013), but distinguishing between these alternatives and $\mathrm{CDM}$ in a robust fashion has proven challenging (e.g., Knebe et al. 2008). The mass scale at which differences between plausible dark matter models is most likely to be evident-at or below the scale of the satellites of the Milky Way (e.g., Anderhalden et al. 2013; Schneider et al. 2014) - is also the mass scale at or below which galaxy formation is inefficient and apparently stochastic (e.g., BoylanKolchin et al. 2011; Power et al. 2014b; Garrison-Kimmel et al. 2016). The latest generation of cosmological hydrodynamical galaxy formation simulations in the CDM model can produce satellite populations that are broadly consistent with observations (e.g., Brooks \& Zolotov 2014; Dutton et al. 2016;
Sawala et al. 2016a, 2016b; Wetzel et al. 2016; Zhu et al. 2016). However, it is noteworthy that the internal properties of galaxies and their satellites in plausible alternatives, such as WDM or Self-interacting Dark Matter, can also provide similarly good consistency with observations (e.g., Zavala et al. 2013; Herpich et al. 2014; Colín et al. 2015; Elbert et al. 2015; Fry et al. 2015; Governato et al. 2015).

These results suggest that, provided a galaxy can form, the properties of the galaxy that we observe are likely to be shaped by the physics of galaxy formation rather than the physics of dark matter (e.g., Herpich et al. 2014). Arguably this is not so surprising, when one considers the relative dominance of baryonic material for the central gravitational potential in all but the most dark matter dominated galaxies, and the relatively short timescales (e.g., crossing times, cooling times) in these regions. What about the outskirts of galaxies, where timescales are long and the influence of dark matter dominates that of baryons? Could these regions offer a test of the underlying dark matter model?

Deep observations of the Milky Way and external galaxies reveal that they are embedded in diffuse, extended stellar structures of shells, streams, and halos (Freeman \& BlandHawthorn 2002; Helmi 2008). These structures are strikingly evident around our nearest massive galactic neighbor, M31see, for example, results from the PAndAS (e.g., McConnachie et al. 2009; Mackey et al. 2010) and SPLASH surveys (e.g., Gilbert et al. 2012) - and they are also apparent in deep imaging of more distant galaxies (e.g., Martínez-Delgado et al. 2010; Radburn-Smith et al. 2011; Monachesi et al. 2013; Trujillo \& Bakos 2013; Crnojević et al. 2015). Stellar halos are predicted to arise naturally in hierarchical galaxy formation models, built up, at least partially, by the merger and accretion events that drive galaxy assembly (e.g., Searle \& Zinn 1978; Helmi \& White 1999; Bullock \& Johnston 2005; Abadi et al. 2006; Johnston et al. 2008; Zolotov et al. 2009; Cooper 
et al. 2010, 2013; Font et al. 2011; Scannapieco et al. 2011; McCarthy et al. 2012); partially, because cosmological galaxy formation simulations have demonstrated that such halos likely have a dual origin, comprising of a component that formed in situ (cf. Abadi et al. 2006; Zolotov et al. 2009; Font et al. 2011), in addition to the accreted component that formed via tidal disruption of satellite galaxies (e.g., Bullock \& Johnston 2005) and star clusters (e.g., Boley et al. 2009). The in situ component is believed to originate in dynamical heating of the stellar disc at early times (cf. McCarthy et al. 2012), and so it dominates at smaller galacto-centric radius; in contrast, the outer halo is dominated by the accreted component (e.g., Zolotov et al. 2009; Scannapieco et al. 2011; Amorisco 2015; Rodriguez-Gomez et al. 2016).

In this paper, we investigate how the underlying dark matter model might influence this accreted component by exploring the spatial and kinematic structure of stellar halos of simulated galaxies, to which we refer hereafter as extended stellar components (ESCs), and focus on those that form in cosmological galaxy formation simulations in CDM and WDM models. We reason that there should be an imprint on properties of the accreted component; because this component is built up through mergers and accretions of lower mass satellite galaxies, it follows that a dark matter model that suppresses the abundance of low-mass subhalos, and consequently low-mass satellite galaxies, is likely to result in less massive - and lower luminosity-ESCs at large galacto-centric radii, or equally, more centrally concentrated ESCs in WDM models compared to the CDM counterpart.

We do not expect the dynamics of subhalos to differ significantly between CDM and WDM models (e.g., Knebe et al. 2008). The orbital distribution will be preferentially radial (Benson 2005), while the orbits of lower mass subhalos and their satellites will require many pericentric passages to decay (Tormen et al. 1998); this implies that tidally stripped stars from a low-mass satellite can be spread over large galactocentric distances, and the greater the number of low-mass satellite undergoing such tidal stripping, the greater the radial extent of the resulting ESC. We make the reasonable assumption that the physical processes that govern galaxy formation (e.g., cooling, star formation, feedback) should not depend on the underlying dark matter, consistent with previous studies (e.g., Herpich et al. 2014; Governato et al. 2015). This means that differences in the properties of the accreted component of the ESC should reflect differences in the abundance of satellites, which depends on the subhalo abundance. These differences could be accessible to future surveys that target the diffuse, low surface brightness environs of galaxies, extending the work of deep imaging studies such as PAndAS (cf. McConnachie et al. 2009) and SPLASH (e.g., Gilbert et al. 2012) and the the "Dragonfly Telephoto Array" (cf. van Dokkum et al. 2014), and so potentially represents an observational test of dark matter.

In the remainder of this paper, we present our exploration of this idea, using cosmological zoom simulations of a set of six Milky Way mass system $\left(M_{200} \simeq 2 \times 10^{1} 2 h^{-1} M_{\odot}\right)$. In all six cases, we follow their formation and evolution in a fiducial CDM model, and for one system we carry out a further set of simulations in three WDM models, with equivalent particle masses of $m_{\mathrm{WDM}}=0.5,1$ and $2 \mathrm{keV} / c^{2}$; we motivate these choices below. All simulations assume the same prescriptions for cooling, star formation and SN feedback, and we carry out a set of simulations to assess the sensitivity of our predictions to the assumed galaxy formation parameters. Details of the simulations are presented in Section 2. Results of our analysis are given in Section 3; here we quantify the $z=0$ spatial and kinematic structure of the ESC; verify that these results are reasonable in the context of the mass assembly histories of the galaxies and the orbital structure of the ESC; and estimate whether or not the differences could provide a useful test of dark matter, allowing for the system-to-system variation we might expect within the CDM model. Finally, in Section 4, we summarize our results.

\section{SIMULATIONS}

Parent Simulation The parent $\Lambda \mathrm{CDM} N$-body simulation is a $256^{3}$ particle $L_{\text {box }}=50 \mathrm{~h}^{-1} \mathrm{Mpc}$ cube. Following Komatsu et al. (2011), we assume total matter, baryon, and dark energy density parameters of $\Omega_{\mathrm{m}}=0.275, \quad \Omega_{\mathrm{b}}=0.0458$, and $\Omega_{\Lambda}=0.725$, and dimensionless Hubble parameter of $h=0.702$, and we compute the CDM power spectrum using CAMB (cf. Lewis et al. 2000), with a power spectrum normalization of $\sigma_{8}=0.816$ and primordial spectral index $n_{\mathrm{s}}=0.968$. This results in a particle mass of $m_{\mathrm{p}} \simeq 5.6 \times 10^{8} h^{-1} M_{\odot}$. The simulation is run with GADGET2 (Springel 2005) with a constant comoving gravitational softening $\epsilon=5 h^{-1} \mathrm{kpc}$, and candidate haloes for resumulation are identified in the friends-of-friends (FOF) group catalog at $z=0$, where we use a linking length of $b=0.2 \bar{d}$. Note that we post-process each FOF group to compute its center of density $\boldsymbol{r}_{\text {cen }}$ (using the iterative "shrinking spheres" method; cf. Power et al. 2003) and obtain virial masses, which we define as

$$
M_{200}=\frac{4 \pi}{3} \times 200 \times \rho_{\text {crit }, 0} R_{200}^{3} ;
$$

here $\rho_{\text {crit }, 0}=2.7755 \times 10^{11} M_{\odot} h^{2} \mathrm{Mpc}^{-3}$ is the critical density of the universe at $z=0$. We select Milky Way mass haloes as those having virial masses of $M_{200} \sim 2 \times 10^{12} h^{-1} M_{\odot}$, which ensures that they are resolved at $z=0$ with $\sim 3500$ particles; this is sufficient to define accurately the Lagrangian region at high redshifts from which the halo collapses.

Galaxy Resimulations We resimulate a suite of 6 Milky Way mass halos (MW01-MW06; see Table 1 for details) with both dark matter and gas. They were chosen to reside in low-density (void) regions, identified using the $\mathrm{V}$-web algorithm of Hoffman et al. (2012), which is a kinematic classification of the cosmic web based on diagonalization of the local velocity shear tensor. Our approach to generating initial conditions is presented in detail in Power et al. (2014a), but we summarize the key steps as follows.

1. We select all particles within radius $5 R_{200}$ at $z=0$ centered on $\boldsymbol{r}_{\text {cen }}$ of the halo of interest within the parent simulation, and use these to define the Lagrangian volume encompassing the desired high resolution region in the resimulation initial conditions at $z=\infty$.

2. We populate this high resolution region with both dark matter and gas particles whose relative mass densities are set by the baryon and dark matter density pararmeters $\Omega_{\mathrm{b}}=0.0458$ and $\Omega_{\mathrm{DM}}=0.2292$, and whose number densities are set by the desired maximum mass resolution of the resimulation. This high resolution region is then embedded within regions of coarser mass resolution consisting of collisionless tidal particles, where the 
Table 1

Galaxy Properties at $z=0$

\begin{tabular}{|c|c|c|c|c|c|c|c|c|c|}
\hline & $\begin{array}{c}M_{200} \\
\left(h^{-1} M_{\odot}\right)\end{array}$ & $f_{\mathrm{g}}$ & $f_{\mathrm{s}}$ & $N_{\mathrm{d}}$ & $N_{\mathrm{g}}$ & $\begin{array}{c}N_{\mathrm{s}} \\
\left(\mathrm{km} \mathrm{s}^{-1}\right)\end{array}$ & $\begin{array}{c}\sigma_{3 \mathrm{D}}^{\mathrm{d}} \\
\left(\mathrm{km} \mathrm{s}^{-1}\right)\end{array}$ & $\begin{array}{c}\sigma_{3 \mathrm{D}}^{\mathrm{g}} \\
\left(\mathrm{km} \mathrm{s}^{-1}\right)\end{array}$ & $\sigma_{3 \mathrm{D}}^{\mathrm{s}}$ \\
\hline MW01 & $2.95 \times 10^{12}$ & 0.095 & 0.077 & 518,571 & 298,102 & 242,495 & 303 & 149 & 410 \\
\hline \multicolumn{10}{|l|}{ MW02 } \\
\hline WDM1 & $2.28 \times 10^{12}$ & 0.098 & 0.079 & 392,963 & 233,625 & 190,266 & 302 & 156 & 327 \\
\hline WDM2 & $2.203 \times 10^{12}$ & 0.095 & 0.077 & 380,905 & 217,819 & 176,575 & 298 & 137 & 324 \\
\hline CDM & $2.26 \times 10^{12}$ & 0.086 & 0.096 & 385,618 & 204,528 & 227,176 & 319 & 134 & 369 \\
\hline MW04 & $2.45 \times 10^{12}$ & 0.097 & 0.08 & 370,748 & 217,678 & 179,171 & 296 & 147 & 383 \\
\hline MW05 & $2.33 \times 10^{12}$ & 0.108 & 0.077 & 383,492 & 253,462 & 167,170 & 298 & 127 & 368 \\
\hline MW06 & $2.54 \times 10^{12}$ & 0.119 & 0.048 & 392,423 & 279,630 & 111,835 & 265 & 151 & 292 \\
\hline
\end{tabular}

Note. For each of the galaxies that form in our runs with fiducial galaxy formation parameters, we give (1) the virial mass, $M_{200}$, as defined in the text; $(2,3) f_{\mathrm{g}}$ and $f_{\mathrm{s}}$, the fraction of $M_{200}$ in gas and stars, respectively; $(3,4,5) N_{\mathrm{d}}, N_{\mathrm{g}}, N_{\mathrm{s}}$, the number of dark matter, gas, and star particles within $R_{200} ;$ and $(6,7,8) \sigma_{3 \mathrm{D}}^{\mathrm{d}}, \sigma_{3 \mathrm{D}}^{\mathrm{g}}$, and $\sigma_{3 \mathrm{D}}^{\mathrm{s}}$, the 3D velocity dispersions of all the dark matter, gas, and star particles within $R_{200}$.

average particle mass increases with increasing distance from the center of the high resolution patch.

3. We choose a starting redshift $z_{\text {start }}$, in this case $z_{\text {start }}=99$, and impose the appropriate set of density perturbations on the composite particle distribution.

The first set are applied to all particles and are the original perturbations used in the parent simulation, with minimum and maximum wavenumbers, $k_{\min }=2 \pi / L_{\text {box }}$ and $k_{\max }=\pi N_{\text {parent }} / L_{\text {box }}$; here $L_{\text {box }}$ is the length of the parent cube and $N_{\text {parent }}$ is the number of particles on a side within this cube.

The second set are applied to the particles in the high resolution region and are a new set of perturbations that were not present in the initial conditions; note that we sample from the appropiate baryon and dark matter power spectra for the gas and dark matter particles separately. Here the minimum and maximum wavenumbers are $k_{\min }=2 \pi / L_{\text {hires }}$ and $k_{\max }=\pi N_{\text {hires }} / L_{\text {hires }}$, where $L_{\text {hires }}$ is the length of the cube encompassing the high resolution patch and $N_{\text {hires }}$ is the number of particles on a side within this cube.

4. Finally, we use the Zel'dovich approximation to generate particles' initial displacements and velocities (cf. Zel'dovich 1970; Efstathiou et al. 1985).

In the case of MW02, we run a series of simulations in which we vary galaxy formation parameters and numerical resolution (see below), and, crucially for this study, the underlying dark matter model. Following Bode et al. (2001), we obtain the initial power spectra for our WDM models by filtering the CDM power spectrum with an additional transfer function of the form

$$
T^{\mathrm{WDM}}(k)=\left(\frac{P^{\mathrm{WDM}}(k)}{P^{\mathrm{CDM}}(k)}\right)^{1 / 2}=\left[1+(\alpha k)^{2 \nu}\right]^{-5 / \nu}
$$

where $\alpha$ is a function of the WDM particle mass (see Equation (A9) of Bode et al. 2001), $k$ is the wave-number and $\nu=1.2$ is a numerical constant. We do not include an additional velocity to mimic the effects of free-streaming in the early universe. Arguably this omission is likely to be unimportant for the WDM particle masses we consider (e.g., Colín et al. 2008; Angulo et al. 2013), but we note also that modeling this effect correctly in a $\mathrm{N}$-body simulation is difficult-it can lead to an unphysical excess of small-scale power in the initial conditions if the simulation is started too early (see Figure 1 of Colín et al. 2008 for a nice illustration of this problem) — so for clarity we ignore this effect (see also discussion in Power 2013).

In running our suite of resimulations, we use a version of GADGET3 that has been extended to model various galaxy formation processes, which we describe briefly in the following subsection. Our version of GADGET3 solves the equations of hydrodynamics using SPHS, a form of SPH that includes a higher order dissipation switch (SPHS; cf. Read \& Hayfield 2012; Hobbs et al. 2013; Power et al. 2014a; Sembolini et al. 2016) with a Wendland $C^{4}$ kernel with 200 neighbors (cf. Dehnen \& Aly 2012). Gravitational force softenings are set using the Power et al. (2003) criterion, $\epsilon_{\mathrm{opt}}=4 R_{200} / \sqrt{N_{200}}$, where $N_{200}$ is the number of particles within $R_{200}$ (cf. Table 1).

Galaxy Formation Prescription We follow the prescriptions for cooling, star formation, and SN feedback set out in Hobbs et al. (2013), which we now describe briefly. Gas cools radiatively at temperatures above $10^{4} \mathrm{~K}$ following Katz et al. (1996), assuming primordial abundances, and between $10^{4}$ and a floor of $T_{\text {floor }}=10^{2} \mathrm{~K}$ following the prescription of Mashchenko et al. (2008). These simulations do not include chemical evolution, which will influence cooling rates as the abundance of heavier elements increases, especially in higher density regions, but we are interested principally in relative differences and adopt the same cooling rates in all of the resimulations.

Gas is prevented from cooling to the point at which the Jeans mass for gravitational collapse becomes unresolved. We quantify mass resolution as $M_{\text {res }}=N_{\text {res }} m_{\text {gas }}$, where $m_{\text {gas }}$ is the mass of a gas particle and $N_{\text {res }}$ is the number of gas particles that correspond to a single resolution element; this is set to $N_{\text {res }}=128$, which is reasonable for our choice of smoothing kernel (for further discussion, see Hobbs et al. 2013). This ensures that the Jeans mass is always resolved within our simulations, and we write the Jeans density as

$$
\rho_{J}=\left(\frac{\pi k T}{\mu m_{\mathrm{p}} G}\right)^{3} M_{\mathrm{res}}^{-2}
$$

where $k$ is Boltzmann's constant, $G$ is the Gravitational constant, $T$ is gas temperature, $m_{\mathrm{p}}$ is the proton mass, and $M_{\text {res }}$ 
is the mass resolution, as defined above. This manifests as a polytropic equation of state $P=A(s) \rho^{4 / 3}$, where $s$ is the entropy; gas is prevented from collapsing to densities higher than given by Equation (3), and any gas that lies on the polytrope forms stars above a fixed density threshold with an efficiency of $\eta=0.1$, in accord with observations of giant molecular clouds (e.g., Lada \& Lada 2003). The star formation rate follows the Schmidt (1959) and Kennicutt (1998) relation, $\rho_{\mathrm{SFR}} \propto \rho_{\text {gas }}^{3 / 2}$, which we implement by employing the dynamical time as the relevant star formation timescale, i.e.,

$$
\frac{d \rho_{*}}{d t}=\eta \frac{\rho_{\mathrm{gas}}}{t_{\mathrm{dyn}}} .
$$

We include feedback from supernovae (SNe) by injecting thermal energy from active star particles into nearby gas particles. Finite resolution implies that each star particle represents a single stellar population, which we assume to have a Salpeter (1955) initial mass function, and they become active once the age of the star particle exceeds the mean main sequence age of stars more massive than $8 M_{\odot}$ and less massive than the maximum value of $100 M_{\odot}$. At that point energy equivalent to $N_{\mathrm{SNe}}$ times the individual $\mathrm{SN}$ energy of $E_{\mathrm{SN}}=10^{51} \mathrm{erg}$ is injected as a delta function in time into a mass of $M_{\text {res }}$ gas particles, thereby ensuring that both star formation and feedback is resolved.

\section{RESULTS}

Visual Impression In Figure 1, we show how the projected density of stellar material within a $50 h^{-1} \mathrm{kpc}$ radius, centered on the galaxy, varies between the fiducial CDM run and its WDM counterparts. In each case, we have rotated the system such that the central galaxy is viewed edge-on in the frame of its principal axes, which we do by diagonalising the reduced moment of inertia tensor computed for star particles within spherical shells,

$$
I_{i j}=\sum \frac{\Delta x_{i} \Delta x_{j}}{r^{2}} .
$$

Here we compute offsets in the three spatial components $\Delta x^{i}$ with respect to the center of density of the mass distribution, projected onto the unit sphere, and then diagonalise to obtain eigenvalues, which provide a measure of the shape of the stellar mass distribution within the shell, and eigenvectors, which provide us with the necessary rotation matrix to convert coordinates into the principal axes. When rotating the system, we use the rotation matrix derived from the innermost shell of particles.

This figure reveals a number of points worthy of note. First, the stellar distribution within the galaxies takes the form of a disc, with a radial scale length that is broadly the same in each of the runs; visually, the CDM disc appears slightly more compact than the corresponding WDM discs. Second, the projected density of extra-disc stellar material is greatest at small galacto-centric radii, and it is flattened in the direction perpendicular to the disc. Third, and most significantly for this study, we observe that the spatial extent of extra-disc material is similar in the CDM and WDM $m_{\mathrm{WDM}}=2 \mathrm{keV} / c^{2}$ runs, but the density appears to decline more rapidly in the WDM $m_{\mathrm{WDM}}=0.5$ and $1 \mathrm{keV} / c^{2}$ runs.
Mass Distribution The flattening evident in Figure 1 is quantified in Figure 2, where we show how the minor-to-major axis ratio $c / a$ varies with radius. The behavior of $c / a$ measured for the different components is broadly similar across the different dark matter models-the dark matter $c / a$ varies little with radius $(\sim 0.8-0.9)$, whereas $c / a$ for both the gas and stars is small at small radii $\left(\sim 0.2-0.4\right.$ at $\left.R \lesssim 0.05-0.1 R_{200}\right)$ before rising rapidly between $\sim 0.1-0.2 R_{200}$ to $c / a \sim 0.7$ for the stellar component and $c / a \sim 0.8-0.9$ for the gas component. This is consistent with our visual impression in Figure 1, at least for the stellar component.

In Figure 3, we focus on the spherically averaged mass density profiles at $z=0$ for the stars, gas, and dark matter (green, blue, and red curves) in the CDM and WDM $m_{\mathrm{WDM}}=2,1$, and $0.5 \mathrm{keV} / c^{2} \mathrm{keV}$ runs (dotted-dashed, dotted, dashed, and solid curves) respectively. Profiles are constructed by defining the center of density $\boldsymbol{r}_{\text {cen }}$ of the composite system using the shrinking spheres method (cf. Power et al. 2003), sorting particles by radius, and assigning them to 25 spherical logarithmic bins equally spaced between $R_{\min }=0.01 R_{200}$ and $R_{200}$.

Interestingly, differences in the dark matter and gas mass profiles are small between dark matter models, especially at radii $R / R_{200} \gtrsim 0.1$. At smaller radii, the spherically averaged gas density differs by a factor of a few in density over a factor of a few in radius within $R / R_{200} \sim 0.1$, but these differences occur in the peripheries of the galaxy disc. However, there are striking differences in the spherically averaged stellar mass density; outside of the region within which the galaxy disc reside, between $R / R_{200} \sim 0.1$ and $R / R_{200} \sim 0.5$, we find that the spherically averaged stellar density is approximately an order of magnitude smaller in the WDM $m_{\mathrm{WDM}}=1$ and $0.5 \mathrm{keV} / c^{2}$ runs than in the CDM and WDM $2 \mathrm{keV} / c^{2}$ runs.

As we demonstrate in appendix Figure 9, we expect these differences in the spherically averaged mass profiles of the ESC to be relatively insensitive to our choice of galaxy parameters parameters - the properties of the central galaxy show a much greater dependence on what we assume for the threshold for star formation $\left(n_{\text {thresh }}\right)$ or strength of SN feedback $\left(\epsilon_{\text {feed }}\right)$. On the other hand, we expect our results to be sensitive to mass resolution-as we resolve lower mass systems and gas can reach higher densities, where star formation occurs and how it is affected by, for example, feedback, will affect when stars form and the rate at which satellites disrupt; this is evident as we go to higher resolution. As we show in Figure 9, this is apparent especially within the central regions where the galaxy resides at $z \simeq 3$ (the latest time at which we we have data available currently), although the properties of the ESC are reasonably consistent between resolutions. However, we focus on relative differences in this study, and we are confident that the runs we use are adequate for this purpose.

To what extent are these differences at $z=0$ evident at earlier times? In Figure 4, we track the growth of the total virial mass, which we define as $M_{200}$ at all redshifts, as well as the gas and stellar masses within $R_{200}$ at each redshift. At late times, the masses in the different components are similar across the models, but as we go to earlier times $(z \gtrsim 4)$, differences between the models become apparent. The rate at which both the total mass (upper curves) and gas mass (middle curves) grows is similar in the CDM, WDM 2 and $1 \mathrm{keV}$ models, but there is a substantial lag in the $0.5 \mathrm{keV}$ model; the rate at which 

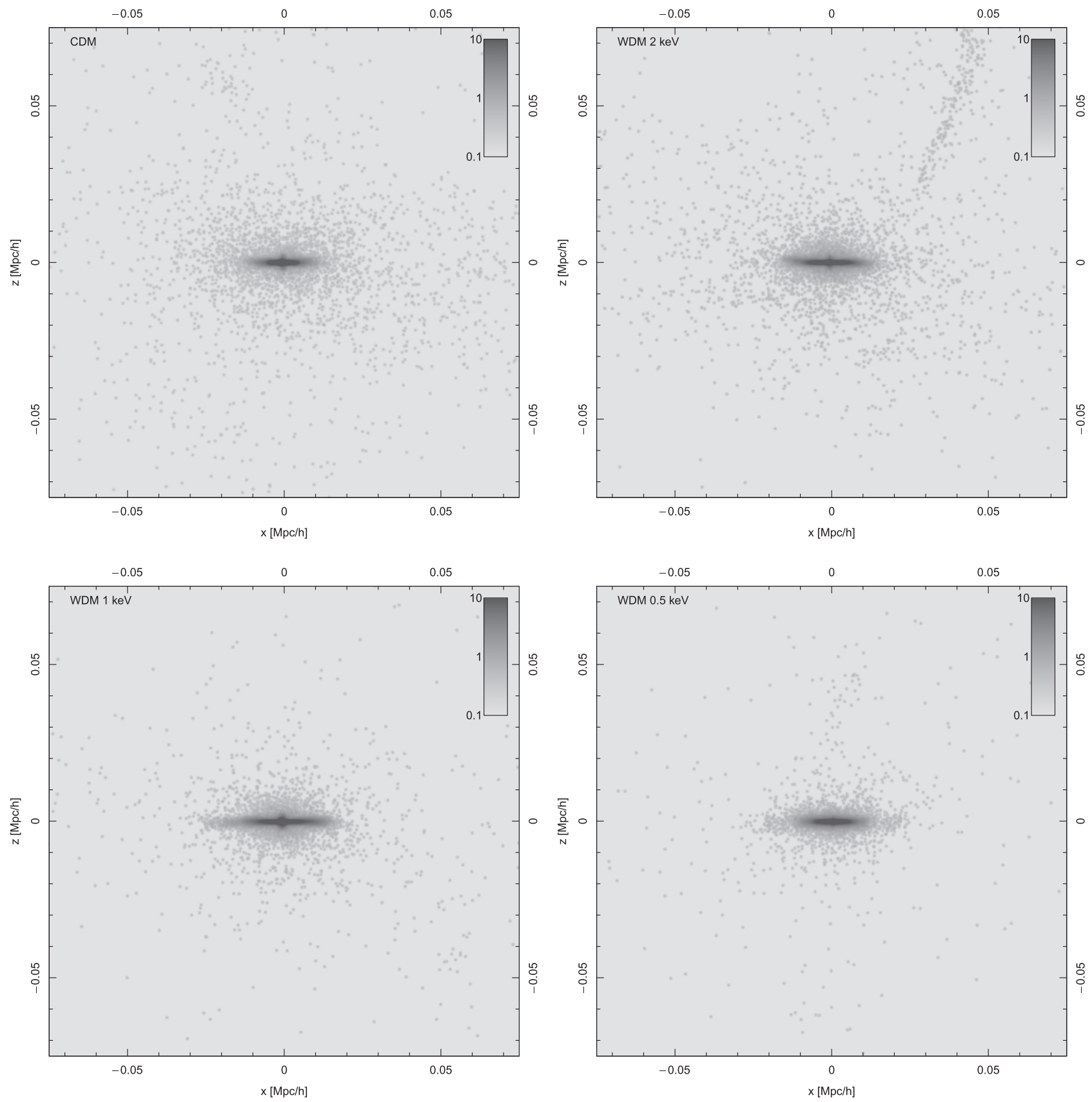

Figure 1. Projected density of stellar material within $50 h^{-1} \mathrm{kpc}$ radius of galaxy at $z=0$. The CDM case is shown in the top left; the WDM $m_{\mathrm{WDM}}=2$, 1 , and $0.5 \mathrm{keV} / c^{2}$ cases are shown in the top right, bottom left and right respectively. In each case, we have rotated the galaxy coordinates such that it is viewed edge on in the frame of the principal axes; see text for further details on how this has been calculated.

the stellar mass grows shows greater variation between the models and is evident down as late as $z \sim 1$.

We explore the growth the stellar mass in more detail in Figure 5, where we separate stellar mass within $R_{200}$ into contributions from $R / R_{200}<0.1$, which contain the central galaxy (upper curves), and $0.1 \leqslant R / R_{200} \leqslant 0.8$ (lower curves). This reveals that, at late times especially, the amount of stellar mass associated with the central galaxy is consistent between the different dark matter runs; the differences that we see in Figure 4 are driven by differences in the outer stellar component surrounding the galaxy. The contribution from the outer stellar component in the CDM model is $\sim 2 \times 10^{9} h^{-1} M_{\odot}$ at $z=0$ and has remained at this level since $z \simeq 4$; the contributions in the WDM 1 and $0.5 \mathrm{keV}$ models are approximately $1 / 2$ and $1 / 4$ of this at $z=0$, but these were larger in the past, by roughly a factor of 2 at $z \simeq 4$.

Kinematics and Orbits Figures 3 and 5 confirm our visual impression that the density of stellar material outside of the galaxy disc drops off more rapidly in the WDM 1 and $0.5 \mathrm{keV}$ runs when compared to the CDM and WDM $2 \mathrm{keV}$ runs. In Figure 6 we investigate whether or not this reflects differences in the kinematics of the stellar material, because the nature of the orbits that a population of stars follow will be imprinted on the mass density profile. We quantify this by considering the ratio of the tangential and radial velocity dispersions, $\sigma_{\text {tan }}$ and $\sigma_{\text {rad }}$, which allow us to estimate the velocity anisotropy as a 


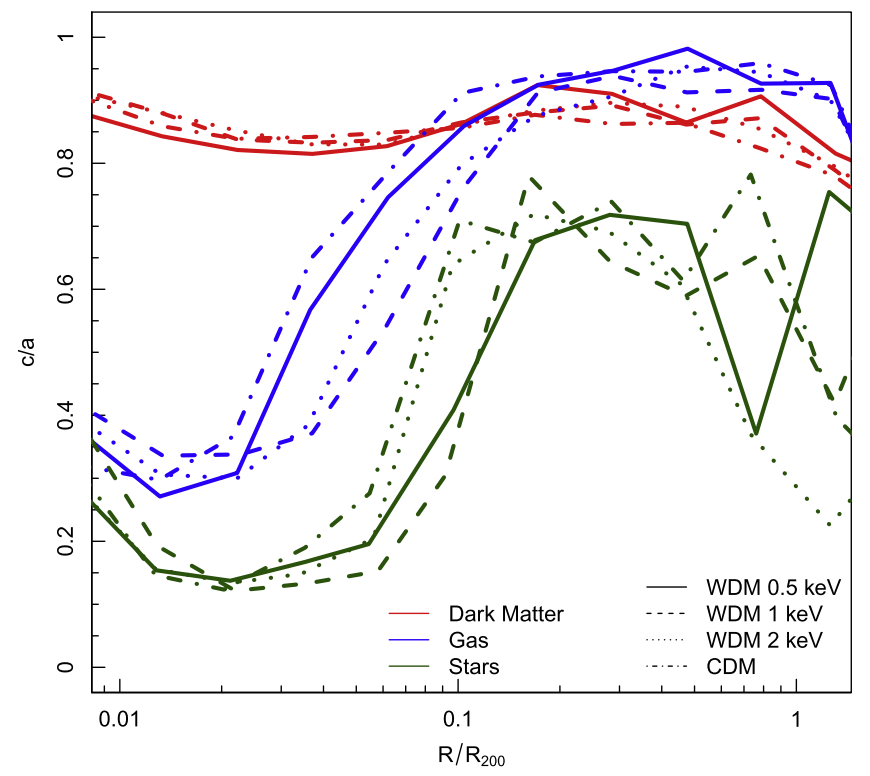

Figure 2. Radial variation of minor-to-major axis $(c / a)$ ratio at $z=0$. We have computed a simple measure of the flattening of the mass distribution, as quantified by $c / a$, as a function of radius (see text for details). Different curves correspond to stellar, gas, and dark matter components (green, blue and red curves) in the fiducial CDM run (dotted-dashed curves) and WDM $m_{\mathrm{WDM}}=2$, 1 , and $0.5 \mathrm{keV} / c^{2}$ curves (dotted, dashed, and solid curves).

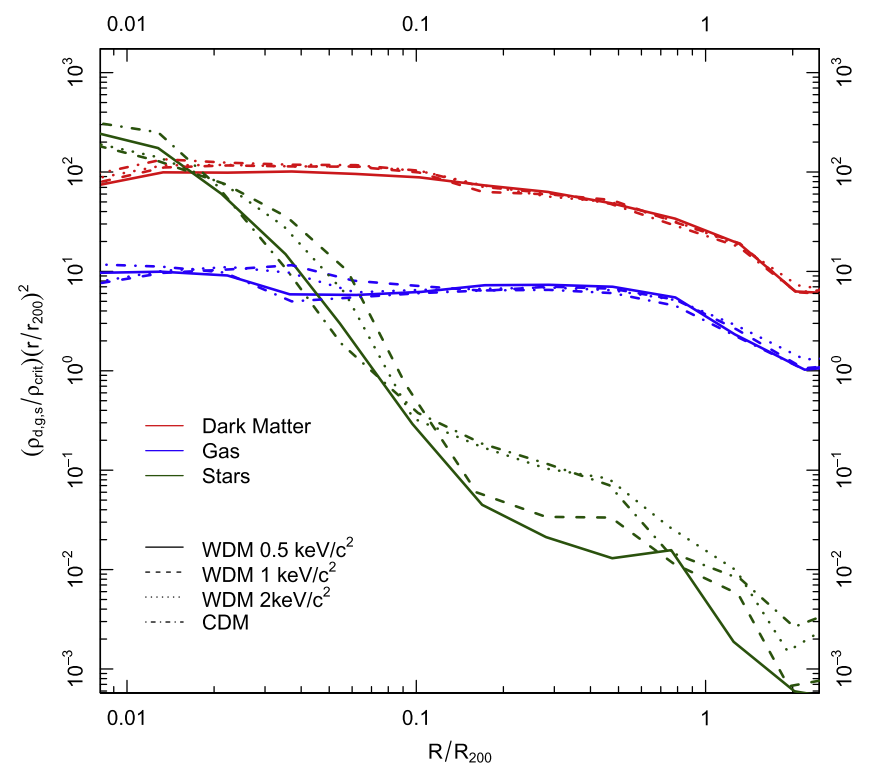

Figure 3. Spherically Averaged Mass Profiles at $z=0$. Here we show spherically averaged stellar, gas, and dark matter mass profiles (green, blue and red curves) in the fiducial CDM run (dotted-dashed curves) and WDM 2, 1 and $0.5 \mathrm{keV}$ curves (dotted, dashed, and solid curves).

function of radius. $\sigma_{\tan } / \sigma_{\mathrm{rad}} \sim 1$ indicates that orbits are approximately isotropic; $\sigma_{\tan } / \sigma_{\text {rad }}<1$ indicates that orbits are preferentially radial; while $\sigma_{\tan } / \sigma_{\text {rad }}>1$ indicates preferentially tangential orbits.

Figure 6 reveals that the velocity anistropy of the dark matter is similar in each of the runs, isotropic at small radii and becoming mildly radial at larger radii. The velocity anisotropy of the gas shows that it is preferentially tangential (unsurprisingly) within $R_{200}$. Between $R / R_{200} \sim 0.3$ and $R / R_{200} \sim 1$, it is mildly tangential and declining with increasing radius, and the behavior is broadly similar between the runs. Within

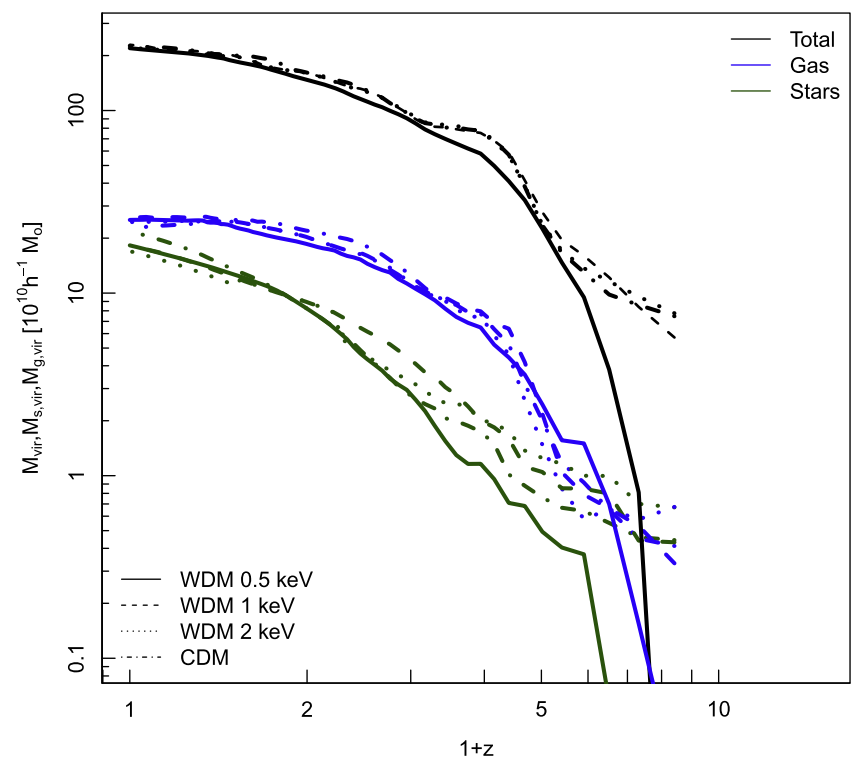

Figure 4. Growth of Virial Mass with Redshift. Here we show how the virial mass $M_{200}$, including stellar, gas, and dark matter mass (black curves), and the stellar and gas masses (green and blue curves resectively) within $R_{200}$ have been assembled as a function of redshift, in the fiducial CDM model (dotteddashed curves) and in the WDM 2, 1 and $0.5 \mathrm{keV}$ models (dotted, dashed, and solid curves respectively).

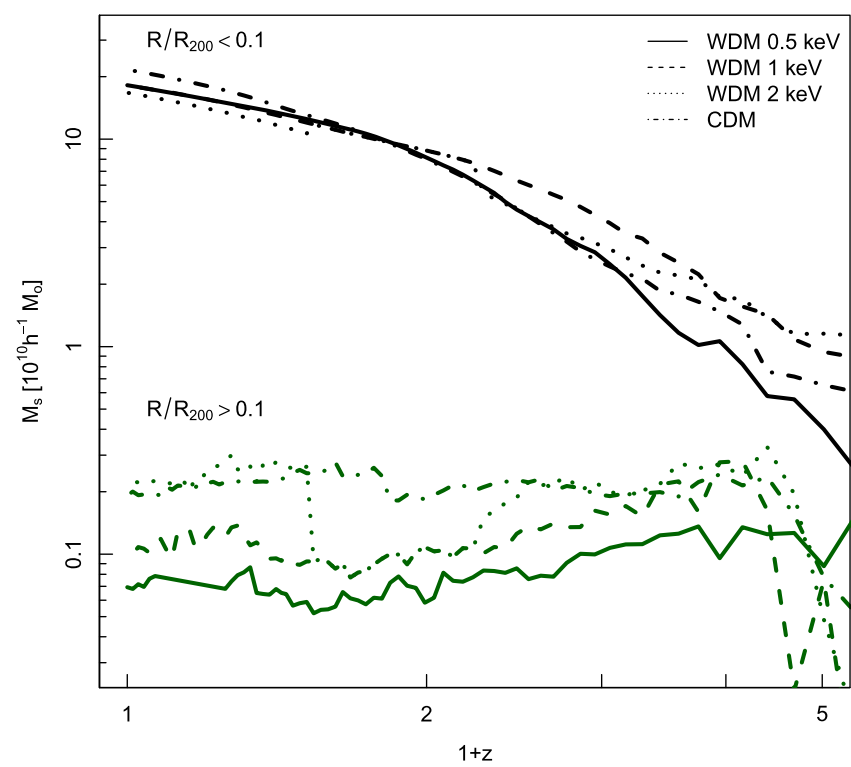

Figure 5. Growth of Stellar Mass in Halo with Redshift. Here we show how the stellar mass in the halo between $0.1 \leqslant R / R_{200} \leqslant 0.8$, in the fiducial CDM model (dotted-dashed curves) and in the WDM 2, 1 and $0.5 \mathrm{keV}$ models (dotted, dashed, and solid curves).

$R / R_{200} \sim 0.3$, the behavior differs sharply and with no obvious trend with underlying dark matter model; the anisotropy peaks with $\sigma_{\tan } / \sigma_{\text {rad }} \sim 3-4$ between $R / R_{200} \sim 0.02-0.1$. The stellar material follows preferentially tangetial orbits within $R / R_{200} \sim 0.1$, as we would expect from the degree of flattening evident in Figure 1, but becomes markedly radial between $R / R_{200} \sim 0.1$ and $R / R_{200} \sim 1$. There are differences within $R / R_{200} \sim 0.1$ between the CDM run and the WDM runs; the anistropy profile peaks and starts to roll over at a smaller radius $\left(R / R_{200} \sim 0.02\right)$ in the CDM run than in the WDM runs $\left(R / R_{200} \sim 0.04\right)$, which reflects the slightly smaller radial scale 


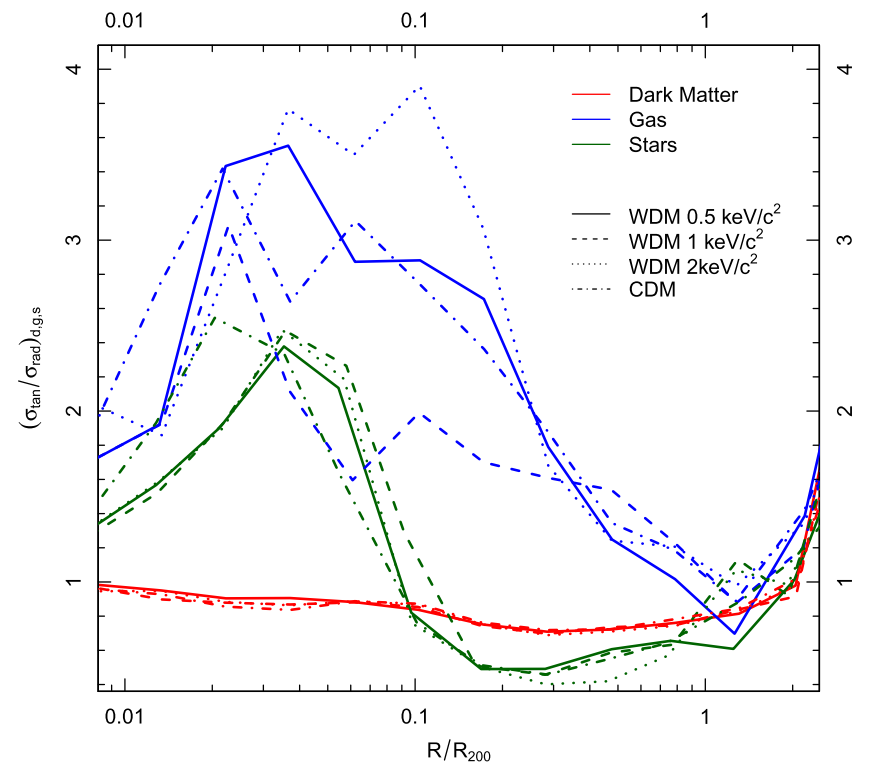

Figure 6. An Estimate of the Velocity Anisotropy at $z=0$. Here we estimate the relative importance of radial to tangential motions by showing the ratio of the spherically averaged stellar, gas, and dark matter tangential and radial velocity dispersions (green, blue, and red curves) in the fiducial CDM (dotteddashed curves) and WDM 2, 1, and $0.5 \mathrm{keV}$ (dotted, dashed, and solid curves) runs.

length we have observed already. At the larger radii of most interest when considering the ESC, however, we find no appreciable differences.

Could differences in the spatial structure of the ESC reflect differences in the kinds of orbits traced out by the progenitors of the material that composes in the ESC? We check this explicitly in Figure 7, where we probe the orbits of the stellar material that lie within the radial range $0.1 \lesssim R / R_{200} \leqslant 1$ at $z=0$. Here we characterize the orbit by $R_{\min }$, the minimum peri-centric distance from the center of the galaxy, estimated from the minimum turning point of the curve tracking star particle radius versus time ${ }^{1}$; star particles that are on their initial infall onto the system lie on the diagonal of $R_{\min }$ versus $R_{0}$, where $R_{0}$ is the present day radius. In this Figure, we smooth the distribution of $R_{\min }$ and $R_{0}$ using a $2 \mathrm{D}$ binned kernel density estimate. Our expectation is that satellite galaxies will follow preferentially radial orbits that bring them close to the center of the potential, and this is borne out by this plot- the majority of the stellar material has values of $R_{\min }$ within a narrow range between $\sim 10$ and $\sim 20 h^{-1} \mathrm{kpc}$. Few of the star particles that constitute the ESC are infalling for the first time, and satellites that have yet to disrupt are apparent as dense knots in the distribution. The key point here is that there is no systematic difference between the distributions in the four models; orbits are preferentially radial (cf. Benson 2005; Abadi et al. 2006; Khochfar \& Burkert 2006; Font et al. 2011; Wetzel 2011; Rashkov et al. 2013; Jiang et al. 2015) and there is no compelling reason why the distribution should differ between CDM and plausible WDM models, as is borne out by numerical simulations (e.g., Knebe et al. 2008)—material funnels from the cosmic web and into the potential of the halo, which does not differ substantially between models.

\footnotetext{
1 Note that this is distinct from pericenter of first infall; this minimum decreases gradually with the number of orbits.
}

A potential test of Dark matter? The results presented so far suggest that the low surface brightness surroundings of galaxies could contain the observable imprint of dark matter. Could, because the differences between the fiducial CDM model and WDM are really only evident in the runs with the more extreme candidates (i.e., $m_{\mathrm{WDM}}<2 \mathrm{keV} / c^{2}$ ). However, for the same reasons that we argued that there should be differences betweenn the CDM model and dark matter models in which the abundance of substructure is suppressed, we can also argue that there should be natural halo-to-halo variation within the CDM model, reflecting variations in assembly histories.

In Figure 8, we show spherically averaged density profiles for six runs in the CDM model (including the run already presented), all with virial masses of $M_{200} \simeq 2 \times 10^{12} h^{-1} M_{\odot}$ at $z=0$ (cf. Table 1), and all selected to lie in low-density environments. This Figure highlights the difficulty of using the ESC as a test of dark matter-the system-to-system variation in the properties of the ESC are as large as we see in the model-tomodel variation when we vary the underlying dark matter model. However, it does suggest that the ESC can be used to extend the concept of galactic archaeology to systems beyond the Milky Way and Andromeda, as is being done in nearby galaxies (e.g., Martínez-Delgado et al. 2010; Radburn-Smith et al. 2011; van Dokkum et al. 2014) and groups (e.g., the PISCeS survey Crnojević et al. 2015), and will become possible for statistical samples of galaxies within the Local Volume with, for example, LSST (cf. LSST Science Collaboration et al. 2009). Combining metallicity, kinematics, and spatial structure, it should be possible to trace the assembly history of galaxies.

\section{SUMMARY}

We have used a set of cosmological zoom galaxy formation simulations of Milky Way mass galaxies, with $M_{200} \simeq 2 \times 10^{12} h^{-1} M_{\odot}$ at $z=0$, to explore whether or not the spatial and kinematic properties of the diffuse, ESCs, in which galaxies are embedded, might depend on the underlying dark matter model. In our simulations of a single system in which we vary the underlying dark matter model, this ESC extends from approximately $15 h^{-1} \mathrm{kpc}$, the outskirts of the galaxy disc, to $R_{200} \simeq 200 h^{-1} \mathrm{kpc}$, and the stellar remnants of disrupting satellite galaxies make a signficant contribution to its mass. For our dark matter models, we considered fiducial CDM and WDM alternatives with particle masses of $m_{\mathrm{WDM}}=0.5,1$, and $2 \mathrm{keV} / c^{2}$; as we discussed in Section 2, although models with $m_{\text {WDM }}<2 \mathrm{keV} / c^{2}$ are not favored by current observational limits, we are interested in establishing whether or not dark matter models that alter the abundance of substructure could leave an imprint on observable properties of galaxies. Because the orbital properties of subhalos in CDM and WDM models are similar, the suppression of low-mass subhalos in WDM models means that the average subhalo mass is more massive than in the corresponding CDM model, which implies shorter merging timescales on average. The tail of low-mass subhalos with long merging timescales in the CDM model means that satellites disrupting in the tidal field of the galaxy lose mass over an extended radial range, tracking their orbit. In other words, the nature of dark matter should be evident in the structure of the ESC, which should be more centrally concentrated in WDM models with lower particle masses (i.e., warmer dark matter). 

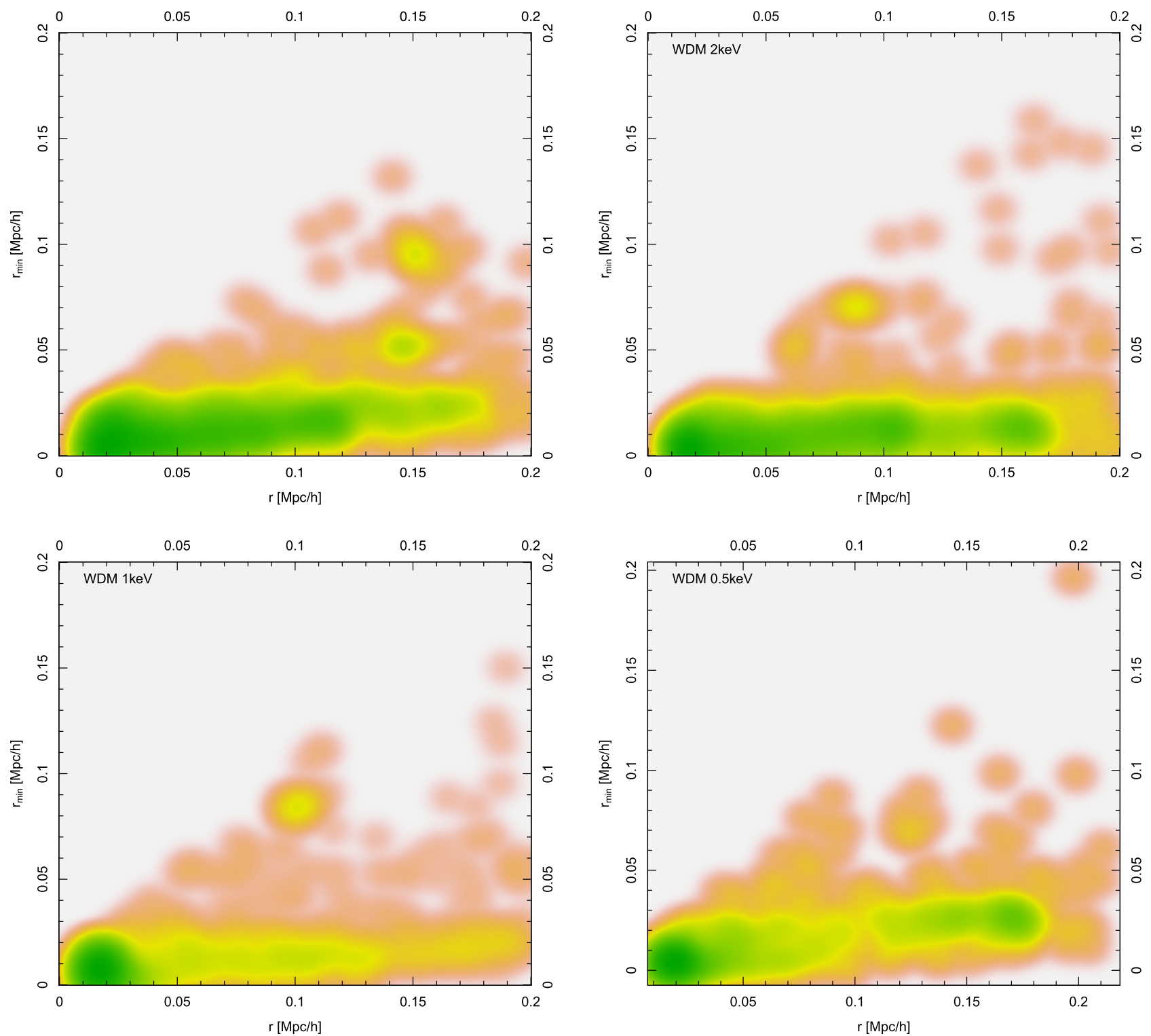

Figure 7. Orbital eccentricities of the extended stellar component. Here we have tracked the orbits of individual star particles and estimated the eccentricity $e$ as the ratio of $r_{\min }$ to $r_{\max }$, where where both are computed following initial pericentric passage. The CDM case is shown in the top left; the WDM $2 \mathrm{keV}, 1 \mathrm{keV}$ and $0.5 \mathrm{keV}$ cases are shown in the top right, bottom left and right respectively.

Our results confirm our expectation that properties of the ESC do vary with the underlying dark matter model, but that differences are most readily apparent only in the more exteme WDM models that we consider, with $m_{\mathrm{WDM}}<2 \mathrm{keV} / c^{2}$. The average stellar density between $15 h^{-1} \mathrm{kpc} \lesssim R \lesssim 150 h^{-1} \mathrm{kpc}$ in the $m_{\mathrm{WDM}}=0.5$ and $1 \mathrm{keV} / c^{2}$ runs is a factor of $\sim 10$ smaller than in the $m_{\mathrm{WDM}}=2 \mathrm{keV} / c^{2}$ and CDM runs. This difference is imprinted early in the history of the galaxy at least back to $z \simeq 4$-and persists to the present day. Interestingly, the properties of the central galaxy, a thin rotationally supported disc, are in good agreement between the different models, consistent with the conclusions of previous studies (e.g., Herpich et al. 2014). Otherwise, the degree of flattening as measured by the axis ratio $c / a$; the kinematics as measured by the ratio of radial to tangential velocity dispersions, $\sigma_{\tan } / \sigma_{\text {rad }} ;$ and the orbits of stars, as estimated from the distribution of pericentric distance $r_{\min }$ to present day position $r_{0}$, are all indistinguishable between models. Similarly, the properties of the dark matter halo and gas are also in very good agreement between models.

Using properties of the ESC to place observational limits on plausible dark matter models that suppress the abundance of low-mass subhalos, and consequently satellites, is likely to be challenging, however. Analyzing the results of a further five zoom simulations of Milky Way mass galaxies, all selected to lie within a similar low-density environment, we find sufficient system-to-system variation in the properties of the spherically averaged stellar density profile beyond the central stellar component-all flattened and rotationally supported - to make observational measurements using spatial structure alone that distinguish between even the more extreme WDM models (i.e., $m_{\mathrm{WDM}}<2 \mathrm{keV} / c^{2}$ ) and CDM unconvincing.

Note that we have focussed essentially on the unresolved ESC-simply considering the spatial and kinematic distribution of stellar material in galactic outskirts, because these should be accessible to deep imaging surveys, possibly 
stacking large numbers of galaxies by central galaxy stellar mass or halo mass bins. It is worth noting that there may be potential to look at the resolved ESC and to combine spatial, kinematic and metallicity substructure information to test dark matter; this will require the kind of statistical sample of Local Volume galaxies that will be accessible with LSST (LSST Science Collaboration et al. 2009). Even if this remains a challenging test of dark matter, there is good reason to expect that we can use properties of the ESC to explore the mass

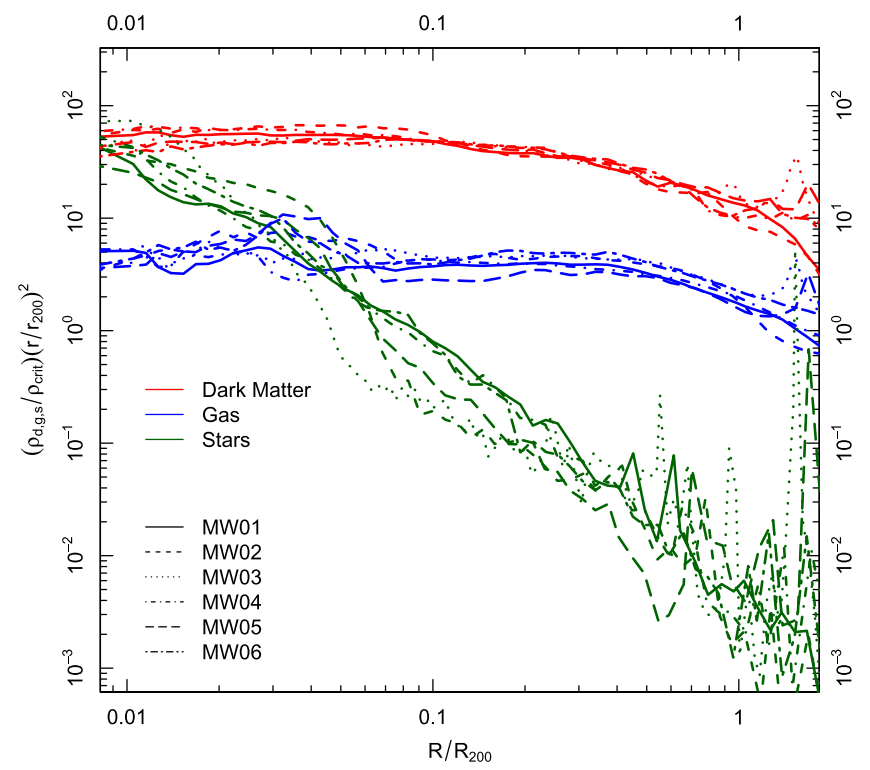

Figure 8. Galaxy-to-galaxy variation in the CDM model. Here we show how much variation between different galaxies we might expect by plotting spherically averaged stellar, gas, and dark matter mass profiles (green, blue and red curves) for the CDM run already analyzed and a further five galaxies, all selected to form in low-density regions with $M_{200} \simeq 2 \times 10^{12} h^{-1} M_{\odot}$ at $z=0$. assembly histories of galaxies, tracing merger and accretion events using material in the outer halo, and placing limits of the growth of galaxies in the context of their larger scale environment. We will explore this idea in forthcoming work.

The authors thank the referee for their careful reading of the paper. CP acknowledges support of Australian Research Council (ARC) Future Fellowship FT130100041 and Discovery Project DP130100117. ASGR acknowledges support of a University of Western Australia Research Fellowship. Both $\mathrm{CP}$ and ASGR acknowledge support of an ARC Discovery Project DP140100198. CP thanks Alexander Hobbs and Justin Read for making this version of GADGET-SPHS available. The research presented in this paper is undertaken as part of the Survey Simulation Pipeline (SSimPL; http://ssimpl.org/). This work was supported by resources provided by the Pawsey Supercomputing Centre with funding from the Australian Government and the Government of Western Australia.

\section{APPENDIX SENSITIVITY TO GALAXY FORMATION PARAMETERS}

Here we explore the extent to which the structure of the ESC, as well as the central stellar, gas, and dark matter components, are sensitive to our choice of galaxy formation parameters. We consider variations in three parameters;

1. the physical threshold density for star formation, $n_{\text {thresh }}$;

2 . the strength of feedback, $\epsilon_{\text {feed }}$; and

3. the temperature floor, $T_{\text {floor }}$, which is the minimum temperature that we allow gas to cool to.

In the first two cases, we consider larger and smaller values of the parameters relative to the fiducial case (i.e., more/less efficient star formation of $n_{\text {thresh }}=0.1 / 100 n_{\text {thresh }}^{\text {fid }}$, strong/no feedback,
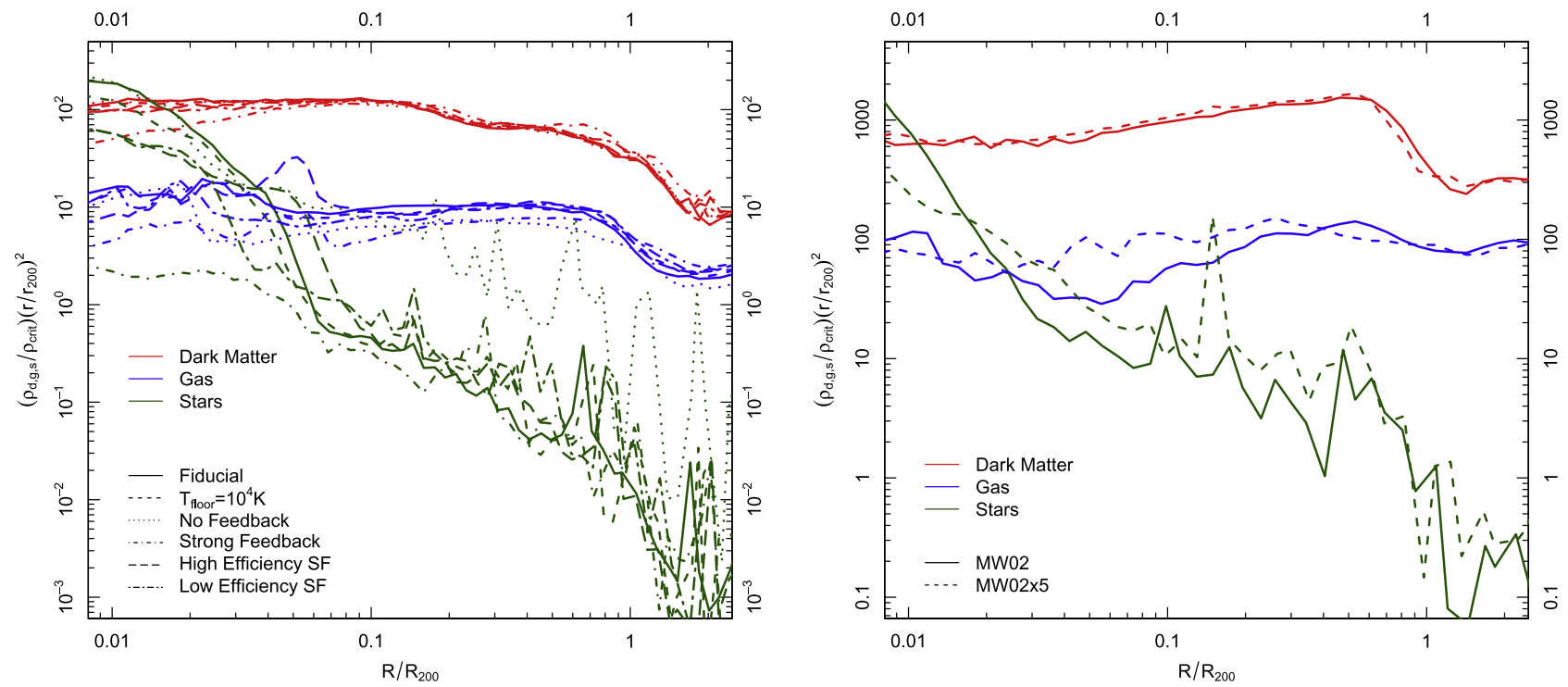

Figure 9. Influence of Galaxy Formation Parameters and Mass Resolution.Left: here we show how our choice of galaxy formation parameters affect the spherically averaged stellar, gas, and dark matter mass profiles (green, blue and red curves) in the galaxy at $z \simeq 1$. Solid curves correspond to our fiducial parameter set, while the dashed curve corresponds to a run with an increased temperature floor of $10^{4} \mathrm{~K}$. The remaining curves indicate runs with strong and no feedback (dotted and dotteddashed), and more and less efficient star formation (long dashed and short-dashed-long dashed. Right: here we show how mass resolution affects spherically averaged stellar, at $z \simeq 3$; fiducial galaxy formation parameters are used. The high resolution run (dashed curves) has a particle mass of $1 / 5$ th that used in the standard resolution runs (solid curves) used in this paper. There are differences in the central stellar density-within the galaxy, such that there is a higher density in the lower resolution run - and in the gas density at intermediate radii, but the shape and amplitude of the stellar density within the ESC is similar between the different resolutions. 
$\left.\epsilon_{\text {feed }}=5 / 0 \epsilon_{\text {feed }}^{\mathrm{fid}}\right)$; in the third, we adopt a temperature floor of $T_{\text {floor }}=10^{4} \mathrm{~K}$, rather than the $100 \mathrm{~K}$. In addition, we compare the results of our standard run, using fiducial galaxy formation parameters, with a particle mass of $1 / 5$ th finer in mass resolution. We show the resulting radial density profiles $\left(\rho r^{2}\right)$ in Figure 9.

In the left hand panel, where we investigate the sensitivity of our results to galaxy formation parameters, the most striking differences arise at small radii, within the galaxy that formsincreasing the temperature floor, or the strength of feedback, results in a drop in central density. Interestingly, varying the threshold for star formation also results in a drop in central density; because $\epsilon_{\text {feed }}$ is kept fixed, the combination of more efficient star formation and feedback compared to inefficient feedback appears to conspire to produce comparable central densities. As far as the properties of the ESC are affected, all of the runs bar "No Feedback" produce mass profiles that are similar - the stellar component has a similar amplitude and shape, while the gas and especially the dark matter components are very similar. In the absence of feedback, the stellar mass density over the radial range $\sim 0.05$ to $\sim 1 R_{200}$ is factor of $\sim 10$ greater than in the other runs. The central stellar and gas densities within the galaxy are similar to the fiducial run, but we note that the central dark matter density is lowered by a factor of a few.

In the right hand panel, we compare results of the system at $z \simeq 3$, the latest time we have available for the higher resolution run currently. Differences in the central stellar density is apparent, with higher densities in the lower resolution run, as we would expect given the relatively coarse resolution of our run (cf. Governato et al. 2010). However, the stellar density over the radial range we are interested in is broadly consistent. It remains to be seen whether or not this holds at $z=0$ and at even higher resolution, but at this point we might expect variations arising from our implementation of the physics of galaxy formation to be as important, if not moreso.

\section{REFERENCES}

Abadi, M. G., Navarro, J. F., \& Steinmetz, M. 2006, MNRAS, 365, 747 Amorisco, N. C. 2015, arXiv:1511.08806

Anderhalden, D., Schneider, A., Macciò, A. V., Diemand, J., \& Bertone, G. 2013, JCAP, 3, 014

Angulo, R. E., Hahn, O., \& Abel, T. 2013, MNRAS, 434, 3337

Angulo, R. E., Lacey, C. G., Baugh, C. M., \& Frenk, C. S. 2009, MNRAS, 399, 983

Benson, A. J. 2005, MNRAS, 358, 551

Benson, A. J., Farahi, A., Cole, S., et al. 2013, MNRAS, 428, 1774

Bode, P., Ostriker, J. P., \& Turok, N. 2001, ApJ, 556, 93

Boley, A. C., Lake, G., Read, J., \& Teyssier, R. 2009, ApJL, 706, L192

Boylan-Kolchin, M., Bullock, J. S., \& Kaplinghat, M. 2011, MNRAS, 415, L40

Brooks, A. M., \& Zolotov, A. 2014, ApJ, 786, 87

Bullock, J. S., \& Johnston, K. V. 2005, ApJ, 635, 931

Colín, P., Avila-Reese, V., González-Samaniego, A., \& Velázquez, H. 2015, ApJ, 803, 28

Colín, P., Valenzuela, O., \& Avila-Reese, V. 2008, ApJ, 673, 203

Cooper, A. P., Cole, S., Frenk, C. S., et al. 2010, MNRAS, 406, 744

Cooper, A. P., D’Souza, R., Kauffmann, G., et al. 2013, MNRAS, 434, 3348

Crnojević, D., Sand, D. J., Spekkens, K., et al. 2015, arXiv:1512.05366

Dehnen, W., \& Aly, H. 2012, MNRAS, 425, 1068

Diemand, J., Kuhlen, M., \& Madau, P. 2007, ApJ, 667, 859

Dutton, A. A., Macciò, A. V., Frings, J., et al. 2016, MNRAS, 457, L74

Efstathiou, G., Davis, M., White, S. D. M., \& Frenk, C. S. 1985, ApJS, 57, 241

Elbert, O. D., Bullock, J. S., Garrison-Kimmel, S., et al. 2015, MNRAS, 453, 29

Font, A. S., McCarthy, I. G., Crain, R. A., et al. 2011, MNRAS, 416, 2802
Freeman, K., \& Bland-Hawthorn, J. 2002, ARA\&A, 40, 487

Fry, A. B., Governato, F., Pontzen, A., et al. 2015, MNRAS, 452, 1468

Gao, L., White, S. D. M., Jenkins, A., Stoehr, F., \& Springel, V. 2004, MNRAS, 355, 819

Garrison-Kimmel, S., Boylan-Kolchin, M., Bullock, J. S., \& Lee, K. 2014, MNRAS, 438, 2578

Garrison-Kimmel, S., Bullock, J. S., Boylan-Kolchin, M., \& Bardwell, E. 2016, arXiv: 1603.04855

Gilbert, K. M., Guhathakurta, P., Beaton, R. L., et al. 2012, ApJ, 760, 76

Governato, F., Brook, C., Mayer, L., et al. 2010, Natur, 463, 203

Governato, F., Weisz, D., Pontzen, A., et al. 2015, MNRAS, 448, 792

Helmi, A. 2008, A\&ARv, 15, 145

Helmi, A., \& White, S. D. M. 1999, MNRAS, 307, 495

Herpich, J., Stinson, G. S., Macciò, A. V., et al. 2014, MNRAS, 437, 293

Hobbs, A., Read, J., Power, C., \& Cole, D. 2013, MNRAS, 434, 1849

Hoffman, Y., Metuki, O., Yepes, G., et al. 2012, MNRAS, 425, 2049

Ishiyama, T., Rieder, S., Makino, J., et al. 2013, ApJ, 767, 146

Jiang, L., Cole, S., Sawala, T., \& Frenk, C. S. 2015, MNRAS, 448, 1674

Johnston, K. V., Bullock, J. S., Sharma, S., et al. 2008, ApJ, 689, 936

Katz, N., Weinberg, D. H., \& Hernquist, L. 1996, ApJS, 105, 19

Kennicutt, R. C., Jr. 1998, ApJ, 498, 541

Khochfar, S., \& Burkert, A. 2006, A\&A, 445, 403

Klypin, A., Kravtsov, A. V., Valenzuela, O., \& Prada, F. 1999, ApJ, 522, 82

Knebe, A., Arnold, B., Power, C., \& Gibson, B. K. 2008, MNRAS, 386, 1029

Komatsu, E., Smith, K. M., Dunkley, J., et al. 2011, ApJS, 192, 18

Lada, C. J., \& Lada, E. A. 2003, ARA\&A, 41, 57

LSST Science Collaboration, Abell, P. A., Allison, J., et al. 2009, arXiv:0912.0201

Lewis, A., Challinor, A., \& Lasenby, A. 2000, ApJ, 538, 473

Mackey, A. D., Ferguson, A. M. N., Irwin, M. J., et al. 2010, ApJL, 717, L11

Martínez-Delgado, D., Gabany, R. J., Crawford, K., et al. 2010, AJ, 140, 962

Mashchenko, S., Wadsley, J., \& Couchman, H. M. P. 2008, Sci, 319, 174

McCarthy, I. G., Font, A. S., Crain, R. A., et al. 2012, MNRAS, 420, 2245

McConnachie, A. W., Irwin, M. J., Ibata, R. A., et al. 2009, Natur, 461, 66

Monachesi, A., Bell, E. F., Radburn-Smith, D. J., et al. 2013, ApJ, 766, 106

Moore, B., Ghigna, S., Governato, F., et al. 1999, ApJL, 524, L19

Pacucci, F., Mesinger, A., \& Haiman, Z. 2013, MNRAS, 435, L53

Power, C. 2013, PASA, 30, 53

Power, C., Navarro, J. F., Jenkins, A., et al. 2003, MNRAS, 338, 14

Power, C., Read, J. I., \& Hobbs, A. 2014a, MNRAS, 440, 3243

Power, C., Wynn, G. A., Robotham, A. S. G., Lewis, G. F., \& Wilkinson, M. I. 2014b, arXiv:1406.7097

Radburn-Smith, D. J., de Jong, R. S., Seth, A. C., et al. 2011, ApJS, 195, 18

Rashkov, V., Pillepich, A., Deason, A. J., et al. 2013, ApJL, 773, L32

Read, J. I., \& Hayfield, T. 2012, MNRAS, 422, 3037

Reed, D., Governato, F., Quinn, T., et al. 2005, MNRAS, 359, 1537

Rodriguez-Gomez, V., Pillepich, A., Sales, L. V., et al. 2016, MNRAS, 458, 2371

Rodriguez-Puebla, A., Behroozi, P., Primack, J., et al. 2016, arXiv:1602.04813

Salpeter, E. E. 1955, ApJ, 121, 161

Sawala, T., Frenk, C. S., Fattahi, A., et al. 2016a, MNRAS, 457, 1931

Sawala, T., Frenk, C. S., Fattahi, A., et al. 2016b, MNRAS, 456, 85

Scannapieco, C., White, S. D. M., Springel, V., \& Tissera, P. B. 2011, MNRAS, 417, 154

Schmidt, M. 1959, ApJ, 129, 243

Schneider, A., Anderhalden, D., Macciò, A. V., \& Diemand, J. 2014, MNRAS, 441, L6

Schneider, A., Smith, R. E., \& Reed, D. 2013, MNRAS, 433, 1573

Searle, L., \& Zinn, R. 1978, ApJ, 225, 357

Sembolini, F., Yepes, G., Pearce, F. R., et al. 2016, MNRAS, 457, 4063

Smith, R. E., \& Markovic, K. 2011, PhRvD, 84, 063507

Springel, V. 2005, MNRAS, 364, 1105

Springel, V., Wang, J., Vogelsberger, M., et al. 2008, MNRAS, 391, 1685

Tormen, G., Diaferio, A., \& Syer, D. 1998, MNRAS, 299, 728

Trujillo, I., \& Bakos, J. 2013, MNRAS, 431, 1121

van Dokkum, P. G., Abraham, R., \& Merritt, A. 2014, ApJL, 782, L24

Wetzel, A. R. 2011, MNRAS, 412, 49

Wetzel, A. R., Hopkins, P. F., Kim, J.-h., et al. 2016, arXiv:1602.05957

Xie, L., \& Gao, L. 2015, MNRAS, 454, 1697

Zavala, J., Vogelsberger, M., \& Walker, M. G. 2013, MNRAS, 431, L20

Zel'dovich, Y. B. 1970, A\&A, 5, 84

Zhu, Q., Marinacci, F., Maji, M., et al. 2016, MNRAS, 458, 1559

Zolotov, A., Willman, B., Brooks, A. M., et al. 2009, ApJ, 702, 1058 